\title{
The Association Between GC Gene Polymorphisms and Metabolic Syndrome in Chinese Rural Population: A Case-Control Study
}

\author{
Tong Zhao \\ Dongdong Zhang \\ Yiming Liu \\ Mingming Feng \\ $\mathrm{Ze} \mathrm{Xu}$ \\ Haoyue Huang \\ Luoya Zhang \\ Wenjie Li \\ Xing Li
}

Department of Nutrition and Food Hygiene, College of Public Health, Zhengzhou University, Zhengzhou, 45000I, Henan, People's Republic of China
Correspondence: Xing Li

Department of Nutrition and Food Hygiene, College of Public Health,

Zhengzhou University, 100 Kexue

Avenue, Zhengzhou, 45000I, Henan,

People's Republic of China

Tel +86 37I 6778 I305

Email lixing530@zzu.edu.cn
Background: $G C$ (group-specific component globulin) encoding VDBP (Vitamin D binding protein) polymorphisms have been associated with susceptibility to some diseases such as diabetes, obesity, osteoporosis, and polycystic ovary syndrome, but the evidence for metabolic syndrome (MetS) in the Chinese rural population is inconclusive. Therefore, we investigated the relationship between $G C$ variants (rs7041, rs4588, rs2282679, and rs705117) and MetS risk as well as VDBP levels in the Chinese rural population.

Patients and Methods: The participants (range of age: 20-90 years) of this case-control study were recruited from the northern Chinese Han rural population. We matched $445 \mathrm{MetS}$ cases with non-MetS controls in a 1:1 ratio by sex, age (within 5 years). Real-time PCR technology was carried out by TaqMan assays to examine the four variants of rs7041, rs 4588, rs2282679, and rs705117 within the $G C$ gene. To identify the association of $G C$ gene polymorphisms with MetS, we calculated ORs using a conditional logistic regression model adjusted for potential confounding factors.

Results: We observed inverse associations of CA and AA genotypes of rs4588 with risk of MetS $(\mathrm{OR}=0.678,95 \%$ CI $0.505-0.910, P=0.010 ; 0.603,95 \%$ CI $0.373-0.973, P=0.039$, respectively) compared with carriers of $\mathrm{CC}$ genotype. A similar relationship was also found between rs2282679 and MetS, showing that carrying AC genotype of rs2282679 can decrease the risk of MetS $(\mathrm{OR}=0.683,95 \%$ CI $0.509-0.917, P=0.011)$ compared with carriers of AA genotype. The results of correlation analysis between MetS components and $G C$ polymorphisms showed that the ORs of AA genotype of rs4588 with high level of TG (triglycerides) and low level of HDL-C (high-density lipoprotein cholesterol) were 0.473 (95\% CI $0.245-0.911, P=0.025)$ and 0.268 (95\% CI $0.117-0.615, P=0.002)$, respectively; the ORs of CC genotype of rs2282679 with high level of TG and low level of HDL-C were 0.428 (95\% CI $0.217-0.842, P=0.014)$ and 0.263 (95\% CI $0.110-0.628, P=0.003)$, respectively. However, there was no significant association between the concentration of VDBP and MetS risk.

Conclusion: Among the Chinese rural population, $G C$ polymorphism was associated with lower metabolic syndrome susceptibility, which might be through affecting blood lipid levels (TG and HDL-C).

Keywords: $G C$, polymorphism, metabolic syndrome, rural population

\section{Introduction}

The metabolic syndrome (MetS) is a cluster of the most dangerous heart attack risk factors: central obesity, raised fasting plasma glucose (FPG), high cholesterol, and elevated blood pressure (BP). ${ }^{1}$ It is estimated that $20-25 \%$ of the global adult 
population have MetS, and they are twice as likely to die from and three times as likely to have a heart attack or stroke than individuals without MetS. ${ }^{2}$ Moreover, populations with MetS have a fivefold higher risk of developing type 2 diabetes mellitus (T2DM). ${ }^{3}$ The underlying causes of MetS remain a challenge to scholars, but both insulin resistance and central obesity have been identified as critical factors. ${ }^{4,5}$ The etiology of MetS and its components involves complex interactions between multiple genetic and environmental factors, such as aging, lack of exercise, proinflammatory states, and hormonal changes. For some individual MetS components, the genetic heritability can be up to $50 \%$ and $13-30 \%$ for collective MetS phenotype. ${ }^{6}$

Increasing evidence indicates that vitamin D deficiency is associated with MetS risk, and the interventions to maintain optimum vitamin D concentrations are considered to be the preventive strategy against MetS. ${ }^{7}$ Some studies have shown that vitamin D affects insulin secretion and sensitivity, and vitamin D deficiency can compromise the capacity of pancreatic $\beta$ cells to convert pro-insulin into insulin, ${ }^{8}$ which further affects the development of MetS. A Mendelian randomization study showed that there was an inverse association between the plasma 25 $(\mathrm{OH}) \mathrm{D}$ concentration and risk of MetS and T2DM in the rural middle-aged and elderly participants. ${ }^{9}$

Vitamin D binding protein (VDBP), also known as group-specific component globulin $(G C)$, is the main carrier of vitamin $\mathrm{D}$ in plasma and plays a crucial role in the development of MetS. Circulating vitamin D released from the skin is bound to VDBP and then transported to adipose tissue, where it is deposited, or to the liver. In the liver, it is hydroxylated by 25 -hydroxylases to 25 -hydroxyvitamin D $(25(\mathrm{OH}) \mathrm{D})$, which can be transported to kidney and subsequently converted to $1,25(\mathrm{OH})_{2} \mathrm{D}$ inside cells. ${ }^{10}$ The $G C$ gene (chromosome 4q13.3; gen ID 2638; MIM 139200; link http://www.ncbi.nlm.nih.gov/gene/2638) encodes the VDBP, which has shown an association with 25(OH)D concentrations in GWAS studies. ${ }^{11}$ Moreover, multiple additional metabolic roles beyond vitamin D transport have been described for VDBP, including binding of fatty acids, actin scavenging, modulation of inflammatory processes and innate immunity, and influencing bone metabolism. ${ }^{12}$

Currently, few studies have focused on the potential relationship between $G C$ polymorphisms and the risk of MetS, especially for the Chinese population. Moreover, the role of $G C$ gene may vary by race. ${ }^{13}$ Therefore, this report aims to assess the associations of $G C$ polymorphisms with MetS and its components as well as VDBP levels by a case-control study in a Chinese rural population.

\section{Methods}

\section{Study Population}

All eligible adult subjects were recruited from the northern Chinese Han rural population from July to August 2013 and from July to August 2015. A total of 445 subjects were diagnosed with MetS based on the new International Diabetes Federation (IDF) definition. ${ }^{2}$ According to the new IDF definition, for one person to be defined as having MetS they must have central obesity (defined as waist circumference (WC) $\geq 90 \mathrm{~cm}$ for Asian men, and $\geq 80 \mathrm{~cm}$ for Asian women) plus any two of the following four factors: 1) raised triglycerides (TG) level: $\geq 150 \mathrm{mg} / \mathrm{dl}$ $(1.7 \mathrm{mmol} / \mathrm{l})$ or specific treatment for this lipid abnormality; 2) reduced high-density lipoprotein-cholesterol (HDLC) $<40 \mathrm{mg} / \mathrm{dl}(1.03 \mathrm{mmol} / \mathrm{l})$ in males and $<50 \mathrm{mg} / \mathrm{dl}(1.29$ $\mathrm{mmol} / \mathrm{l}$ ) in females or specific treatment for this lipid abnormality; 3) raised BP: systolic $\mathrm{BP} \geq 130$ or diastolic $\mathrm{BP} \geq 85 \mathrm{~mm} \mathrm{Hg}$ or treatment of previously diagnosed hypertension; 4) raised FPG; FPG $\geq 100 \mathrm{mg} / \mathrm{dl} \quad(5.6$ $\mathrm{mmol} / \mathrm{l}$ ) or previously diagnosed T2DM. Each MetS case was matched individually with a non-MetS control by the same gender, similar age (within 5 years).

\section{Data Collection and Laboratory Measurements}

All participants completed a standard questionnaire (including gender, age, smoking situation, drinking situation, family history of chronic disease, and medication history of lipid-lowering drugs, antihypertensive drugs, and antidiabetic agents) and underwent a physical examination (body weight, height, hip circumference, WC, systolic BP, and diastolic BP). A family history of disease (T2DM, hyperlipidemia, obesity, and hypertension) was assessed by asking whether participants had a first-degree family member diagnosed with disease. ${ }^{14}$ Participants who smoked $\geq 100$ cigarettes during their lifetime were classified as smokers. ${ }^{15}$ Alcohol consumption was defined as having consumed alcohol $\geq 12$ times in the last year. ${ }^{15} \mathrm{BP}$ was measured three times by using an electronic sphygmomanometer (Omron, HEM-770AFuzzy, Kyoto, Japan) after at least a 5-min rest, with participants in a seated position. The average of the three measurements was used for analysis. ${ }^{16}$ Fasting blood samples (overnight fasting $\geq 8$ 
h) were collected in a vacuum tube. After anticoagulation, plasma was centrifuged (3000 rpm for 10 minutes) and stored at $-80^{\circ} \mathrm{C}$ for biochemical determination. FPG, TG, TC (total cholesterol), and HDL-C were tested by the automatic biochemical analyzer (KHB360, Shanghai, China). LDL-C (low-density lipoprotein cholesterol) level was estimated by Friedewald formula. ${ }^{17}$ The VDBP concentrations were detected by ELISA kits (Sangon Biotech, Shanghai, PR China). All assessments were done in the same lab.

\section{Single Nucleotide Polymorphism (SNP) Selection, DNA Extraction and Genotyping}

Based on an extensive literature review and information from the HapMap and NCBI databases, we selected four related SNPs (rs7041, rs4588, rs2282679, and rs705117). The selection criteria were the minor allele frequency $(\mathrm{MAF})>0.01$ and location in significant gene functional regions such as gene promoter, exon and intron regions. The detailed information of the four SNPs is shown in Table 1.

Genomic DNA was extracted from EDTA-treated whole blood using a DNA extraction kit (DNA blood kit, Bioteke, Beijing, China) following the procedure detailed in the kit. The SNP loci were genotyped by TaqMan SNP genotyping reagents purchased from Applied Biosystems on a 7500 Fast real-time quantitative fluorescence PCR instrument. Ten percent of samples underwent repeat genotyping to ensure genotyping reproducibility, and the concordance rate was $99.5 \%$. All assess were done in the same lab.

\section{Sample Size and Data Analysis}

Preliminary data to conduct formal power analyses were not available when the study was designed. However, we used previous studies of $G C$ variants and MetS components ${ }^{18,19}$ to obtain a rough estimate of the relative

Table I Information on the Selected SNPs in GC Gene

\begin{tabular}{|l|l|l|l|l|}
\hline SNPs & Allele & Position & Location & MAF $^{\mathbf{a}}$ \\
\hline rs704I & T>G & $4: 71752617$ & Exon & 0.382 \\
rs4588 & C>A & $4: 71752606$ & Exon & 0.208 \\
rs2282679 & A $>$ C & $4: 71742666$ & Intron & 0.202 \\
rs705II7 & G>A & $4: 71742398$ & Intron & 0.422 \\
\hline
\end{tabular}

Note: All SNP information from NCBI database GRCh38. p7.

Abbreviation: SNP, Single nucleotide polymorphism; ${ }^{\mathrm{a}} \mathrm{MAF}$, minor allele frequencies based on 1000 Genomes Project. risk $(\mathrm{OR}=0.60)$ and consider the MAF (minor allele frequencies) of rs2282679 as the prevalence of risk factor $(P=0.202)$. Based on this, we estimated a required sample size of 445 per group to detect an effect of similar magnitude at an $\alpha$ level of 0.05 with $80.2 \%$ statistical power.

Categorical variables were shown as numbers (percentages) and were analyzed by chi-square test. Continuous variables were expressed as medians with corresponding interquartile ranges for data with skewed distribution. Independent sample $t$-tests were used for comparisons of continuous variables, and they were log-transformed before analysis if the variables did not conform to a normal distribution. For variables not conform to a normal distribution after log-transformation, we analyzed them using the Wilcoxon rank sum test. The frequency distribution of genotypes and allele was checked for cases and controls, and deviation from the HardyWeinberg equilibrium was assessed in all objects by Fisher's exact test/Pearson's Chi-square test. We used a conditional logistic regression model to estimate ORs and corresponding 95\% CIs for the relationship between SNPs of the $G C$ gene and MetS with its components, adjusting for smoking status, drinking status, family history of diabetes, family history of hypertension, family history of hyperlipidemia, and family history of obesity. Associations between MetS and VDBP levels were determined by the conditional logistic regression model after controlling the confounding factors. Range for levels of VDBP was defined as follows: VDBP-L $(<300 \mu \mathrm{g} / \mathrm{mL})$; VDBP-M $\quad(300-600 \mu \mathrm{g} / \mathrm{mL}) ; \quad$ VDBP-H $\quad(>600 \mu \mathrm{g} / \mathrm{mL}) .{ }^{20}$ Moreover, we conducted a Kruskal-Wallis $H$-test to estimate the relationships between $G C$ gene variants and the concentration of VDBP. All reported $P$ values were twosided and were considered statistically significant at $P<$ 0.05. All statistical analyses were performed using SAS 9.4 (SAS Inst. Inc., Cary, NC, USA).

\section{Results}

\section{Population Characteristics}

Characteristics of all subjects $(n=890)$ in this study are shown in Table 2. There was no significant difference between the MetS and control groups in the level of smoking status, drinking status, family history of these four chronic diseases, LDL-C, and VDBP $(P=0.237$, $1.000,0.426,0.214,0.306,0.194,0.080,0.735$, respectively). However, compared with the control group, cases of MetS display higher levels of BMI, WC, FPG, TC, TG SBP, DBP, and lower levels of HDL-C $(P<0.05)$. 
Table 2 Anthropometric and Clinical Characteristics of Study Participants

\begin{tabular}{|c|c|c|c|}
\hline Characteristics & MetS $(n=445)$ & Control $(n=445)$ & $P$ \\
\hline Male & $194(43.60 \%)$ & $194(43.60 \%)$ & I \\
\hline Age (years) & $56.00(46.00-66.00)$ & $55.00(46.00-65.00)$ & 0.963 \\
\hline Smoking & $98(22.02 \%)$ & $113(25.39 \%)$ & 0.237 \\
\hline Drinking & $60(13.48 \%)$ & $60(13.48 \%)$ & I \\
\hline Family history of diabetes & $99(22.25 \%)$ & $84(18.88 \%)$ & 0.426 \\
\hline Family history of hyperlipidemia & $39(8.76 \%)$ & $26(5.84 \%)$ & 0.214 \\
\hline Family history of obesity & $16(\%) 3.60$ & $9(2.02 \%)$ & 0.306 \\
\hline Family history of hypertension & $177(39.78 \%)$ & $156(35.06 \%)$ & 0.194 \\
\hline BMI $\left(\mathrm{kg} / \mathrm{m}^{2}\right)$ & $27.63(25.93-29.79)$ & $24.30(22.09-26.59)$ & $<0.01$ \\
\hline WC $(\mathrm{cm})$ & $95.00(90.00-100.00)$ & $84.00(77.00-90.00)$ & $<0.01$ \\
\hline FPG (mg/dl) & $5.44(4.57-7.17)$ & $4.64(4.07-5.17)$ & $<0.01$ \\
\hline TC $(\mathrm{mg} / \mathrm{dl})$ & $4.51(3.95-5.28)$ & $4.43(3.90-5.13)$ & 0.015 \\
\hline TG (mg/dl) & $1.97(1.36-2.68)$ & $1.06(0.7 I-1.46)$ & $<0.01$ \\
\hline HDL-C (mg/dl) & $1.21(1.03-1.39)$ & $1.46(1.28-1.65)$ & $<0.01$ \\
\hline LDL-C (mg/dl) & $2.33(1.90-3.03)$ & $2.49(2.04-2.99)$ & 0.080 \\
\hline $\mathrm{SBP}(\mathrm{mm} \mathrm{Hg})$ & $133.00(123.00-148.00)$ & $123(1 \mid 5.00-137.00)$ & $<0.01$ \\
\hline $\mathrm{DBP}(\mathrm{mm} \mathrm{Hg})$ & $85.00(80.00-92.00)$ & $80.00(75.00-88.00)$ & $<0.01$ \\
\hline $\operatorname{VDBP}(\mu \mathrm{g} / \mathrm{mL})$ & $136.40(103.99-284.79)$ & $145.97(100.88-336.99)$ & 0.735 \\
\hline
\end{tabular}

Note: Data are displayed as medians (interquartile range) or numbers (\%).

Abbreviations: BMI, body mass index; WC, waist circumference; FPG, fasting plasma glucose; TC, total cholesterol; TG, triglyceride; HDL-C, high-density lipoprotein cholesterol; LDL-C, low-density lipoprotein cholesterol; SBP, systolic blood pressure; DBP, diastolic blood pressure; VDBP, vitamin D binding protein.

\section{Association of GC Gene and MetS}

The genotype distributions of $G C$ polymorphisms obeyed Hardy-Weinberg equilibrium in all participants $(P=0.245$, $0.676,0.164$ and 0.685 for rs 7041, rs4588, rs2282679, and rs705117, respectively). The distributions for alleles and genotypes of the four SNPs in the two groups are presented in Figure 1. Similar genotype frequencies were observed between the control and MetS groups for rs7041 and rs705117 (Figure 1A and D), with $P$-values of 0.431 and 0.142 , respectively. The $\operatorname{rs} 7041(\mathrm{~T}>\mathrm{G})$ and rs705117 (G>A) polymorphisms did not show any allelic association with MetS, $P=0.216$ and 0.056 , respectively. However, the distributions of rs4588 and rs2282679 were meaningfully different $(P=0.012$ and 0.021 , respectively, Figure $1 \mathrm{~B}$ and $\mathrm{C})$. The $\mathrm{CA}$ and $\mathrm{AA}$ genotype frequencies of rs4588 in controls were significantly higher than in the MetS group, and the AA genotype frequency of rs2282679 in controls was lower than in the MetS group. The rs4588 $(\mathrm{C}>\mathrm{A})$ and $\mathrm{rs} 2282679(\mathrm{~A}>\mathrm{C})$ polymorphisms showed allelic association with MetS, $P=0.005$ and 0.009, respectively.

The associations of $G C$ polymorphisms (rs7041, rs4588, rs2282679, and rs705117) with MetS risk susceptibility are shown in Table 3. Conditional logistic regression model demonstrated that $G C$ polymorphisms were associated with MetS risk after adjusting for smoking status, drinking status, and family history of chronic diseases (T2DM, hypertension, hyperlipidemia, and obesity). Carriers of the CA and AA genotypes of rs4588 have lower MetS risk than those carrying the CC genotype, with the adjusted ORs (95\% CI) being 0.678 (0.505$0.910, P=0.010)$ and $0.603(0.373-0.973, P=0.039)$, respectively. Likewise, participants with $\mathrm{AC}$ and $\mathrm{AC}+\mathrm{CC}$ genotypes of rs2282679 were less likely to develop MetS than those carrying the AA genotype, and the ORs (95\% CI) were $0.683(0.509-0.917, P=0.011)$ and 0.683 (0.519-0.897, $P=0.006)$, respectively. However, no statistically significant relationships were detected between genotypes and MetS risk for rs7041 and rs705117.

\section{Relationship Between GC Gene and Metabolic Syndrome Components}

According to the new IDF definition, there are six components of metabolic syndrome, including TG, HDL-C, systolic BP, diastolic BP, FPG, and WC. The associations of $G C$ polymorphisms and components of MetS are exhibited in Figure 2. The variants of rs4588, rs2282679 and rs705117 were associated with TG and HDL-C, respectively (Figure 2A and B). Compared with $\mathrm{CC}$ and $\mathrm{CA}$ genotypes of rs4588, the population carrying mutant homozygote AA tended to have normal $\mathrm{TG}(\mathrm{OR}=0.512$, $95 \%$ CI $0.270-0.970, P=0.025)$ and HDL-C $(\mathrm{OR}=0.338$, 

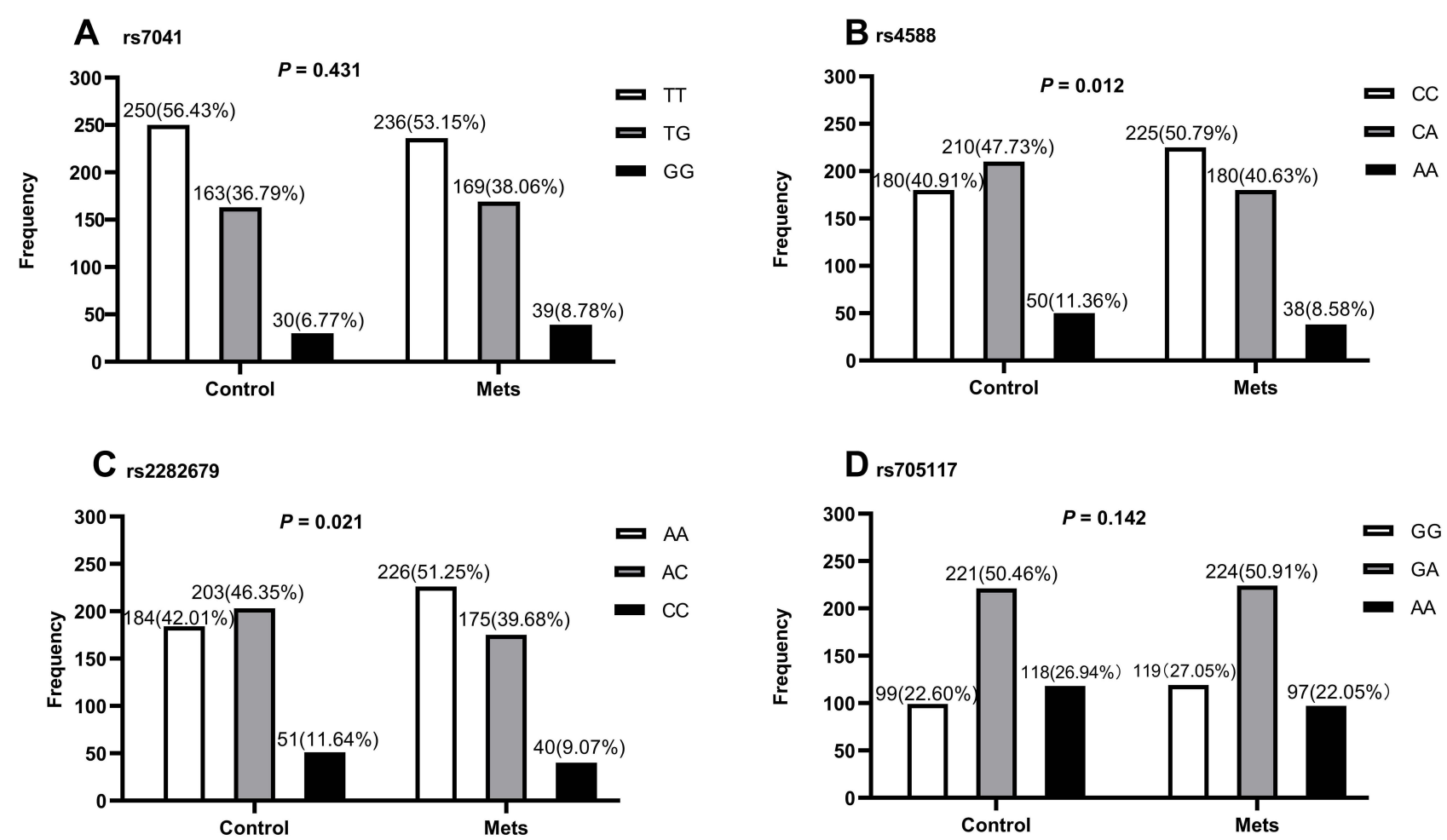

Figure I Genotypic distributions of single nucleotide polymorphisms in the GC gene among MetS patients and controls. The total number in each group is not equal to 445 because of failed genotyping for some samples. Data are presented as count numbers and percentages. (A) Frequencies of rs704I, (B) frequencies of rs 4588 , (C) frequencies of rs2282679, and (D) frequencies of rs705I I7. The $P$-values were generated by Pearson's $\chi^{2}$ tests.

95\% CI 0.154-0.741, $P=0.002$ ) levels in plasma. Carriers of the CC genotype of rs2282679 were more likely to have normal TG and HDL-C levels in plasma, compared with AA and CA genotypes of rs2282679 (OR $=0.456[0.238$ $0.875]$ and $0.304[0.134-0.692]$, respectively, $P<0.05)$. For rs705117, subjects carrying the AA genotype tended to have normal HDL-C concentrations compared to those carrying the GG genotype $(\mathrm{OR}=0.498,95 \%$ CI $0.277-$ $0.984, P=0.020)$. Significantly, compared with $\mathrm{GC}$ and AA genotypes of rs705117, carriers of GG genotype tend to have high DBP level (Figure 2D). However, there were no significant associations between $G C$ variants and SBP, FPG and WC (Figure 2C, E and F).

\section{Associations Between VDBP Level and Metabolic Syndrome}

We detected the associations between $G C$ polymorphism and the concentration of VDBP (Table 4). VDBP levels were significantly different among three genotypes of rs2282679 $(P=0.042)$. There was marginal significance in the associations of VDBP levels and rs4588 variants $(P=0.052)$. Meanwhile, we conducted a conditional logistic regression analysis to evaluate the associations between VDBP levels and MetS; however, no significant association was found between them (Table 5).

\section{Discussion}

It has been shown by our results that the carriers of $\mathrm{CA}$ +AA genotypes of rs4588 and AC genotype of rs2282679 tend to have a reduced metabolic syndrome susceptibility in the Chinese rural population. Meanwhile, according to the analyses of MetS components, significant negative correlations were found between AA genotype of rs4588 and CC genotype of rs2282679 in $G C$ gene and the plasma contents of TG and HDL-C, whereas differential expressions of plasma VDBP had no significant effect on the risk of MetS.

Our findings provide reliable evidence that the polymorphisms of $G C$ gene are correlated with MetS risk in Chinese population. Nevertheless, the findings of relevant studies remain inconsistent. For instance, a study based on the Thailand population found that male subjects with the CA genotype for rs4588 had an increased risk of MetS compared to those with the CC wild-type, ${ }^{19}$ which is contrary to the conclusion of the present research. This 
Table 3 Associations of Genotypes of GC Gene and Risk of Metabolic Syndrome in Han Chinese

\begin{tabular}{|c|c|c|c|c|}
\hline SNPs & Genotypes & Adjusted ORs ${ }^{a}$ & $95 \% \mathrm{Cl}$ & $P$ \\
\hline \multirow[t]{5}{*}{ rs704l } & TT & 1 & & \\
\hline & TG & 1.103 & $0.830-1.465$ & 0.501 \\
\hline & GG & 1.367 & $0.807-2.313$ & 0.245 \\
\hline & $\mathrm{TG}+\mathrm{GG} / \mathrm{TT}$ & 1.142 & $0.870-1.479$ & 0.339 \\
\hline & $\mathrm{GG} / \mathrm{TT}+\mathrm{TG}$ & 1.310 & $0.785-2.186$ & 0.301 \\
\hline \multirow[t]{5}{*}{ rs4588 } & $\mathrm{CC}$ & I & & \\
\hline & $\mathrm{CA}$ & 0.678 & $0.505-0.910$ & 0.010 \\
\hline & $\mathrm{AA}$ & 0.603 & $0.373-0.973$ & 0.039 \\
\hline & $C A+A A / C C$ & 0.663 & $0.503-0.875$ & 0.004 \\
\hline & $\mathrm{AA} / \mathrm{CC}+\mathrm{CA}$ & 0.751 & $0.478-1.178$ & 0.212 \\
\hline \multirow[t]{5}{*}{ rs2282679 } & AA & 1 & & \\
\hline & $A C$ & 0.683 & $0.509-0.917$ & 0.011 \\
\hline & $\mathrm{CC}$ & 0.668 & $0.416-1.073$ & 0.095 \\
\hline & $A C+C C / A A$ & 0.683 & $0.519-0.897$ & 0.006 \\
\hline & $C C / A A+A C$ & 0.774 & $0.494-1.214$ & 0.264 \\
\hline \multirow[t]{5}{*}{ rs705II7 } & GG & I & & \\
\hline & GA & 0.821 & $0.580-1.162$ & 0.265 \\
\hline & AA & 0.689 & $0.470-1.008$ & 0.055 \\
\hline & $\mathrm{GA}+\mathrm{AA} / \mathrm{GG}$ & 0.769 & $0.560-1.005$ & 0.103 \\
\hline & $\mathrm{AA} / \mathrm{GG}+\mathrm{GA}$ & $0.78 I$ & $0.57 \mid-1.068$ & 0.122 \\
\hline
\end{tabular}

Note: ${ }^{a}$ Adjusted for smoking status, drinking status, family history of diabetes, family history of hypertension, family history of hyperlipidemia, and family history of obesity. Abbreviations: OR, odds ratio; SNP, single nucleotide polymorphism.

may be due to the unique genetic backgrounds of each ethnic population, as well as the fact that the replication for findings of complex diseases such as MetS has been relatively poor. ${ }^{21}$ Moreover, variations in the allele frequency of Chinese and Thailand's populations may partly explain the discrepancy, as the allele frequency of rs 4588 in Chinese differs dramatically from other populations, when assessed by the 1000 Genomes Project Phase 3 sequence data in NCBI $(\mathrm{C}=0.739$ in East Asian; $\mathrm{C}=$ 0.697 in South Asian). In the meantime, correlation analysis has only focused on the relationship of vitamin D levels and $G C$ gene polymorphisms ${ }^{22,23}$ or between vitamin D contents and MetS. ${ }^{24}$ Future studies with much more cases are needed to confirm the associations between $G C$ gene variants and MetS risk among various populations.

In addition, we found that certain $G C$ variants were associated with a reduced risk of MetS, as well as low levels of TG and high HDL-C. Hence, it is speculated that the $G C$ may modulate the susceptibility to MetS by affecting the lipid profiles, and the findings of several reports were in favor of this speculation. The rs4588 and rs2282679 are located in the exon and intron of the GC gene, respectively. Variations in rs4588 and rs 2282679 may result in deleterious effects on precursor mRNA splicing, as witnessed by aberrant expression of alternatively spliced transcripts and a tendency to develop certain diseases. ${ }^{25}$ Though no study has yet directly demonstrated the causal relation of $G C$ variants and lipid profiles, Grave et al found that $R X R G$ rs2134095 and $G C$ rs7041 exerted a synergistic effect on reducing LDL-C levels, ${ }^{26}$ while a remarkable correlation between dyslipidemia and $G C$ rs2282679 was illustrated by Foucan et al. ${ }^{27}$ Meanwhile, a prospective cohort study found that $G C$ gene variants were linked with lipid metabolism and the impact was mediated, at least partially through dietary intake. ${ }^{28}$ Moreover, many previous studies have indicated that hypertriglyceridemia is strongly associated with MetS components. ${ }^{29-31}$ It was shown by Sesso et al with a 10.8 years of follow-up study that HDL-C levels were inversely associated with risk of hypertension. ${ }^{32}$ Additionally, there is a clear association among HDL-C and diabetes and obesity. ${ }^{33,34}$ In consequence, the combination of low HDL-C content with elevated TG level accounts for a critical factor for the development of MetS. $^{35}$ 

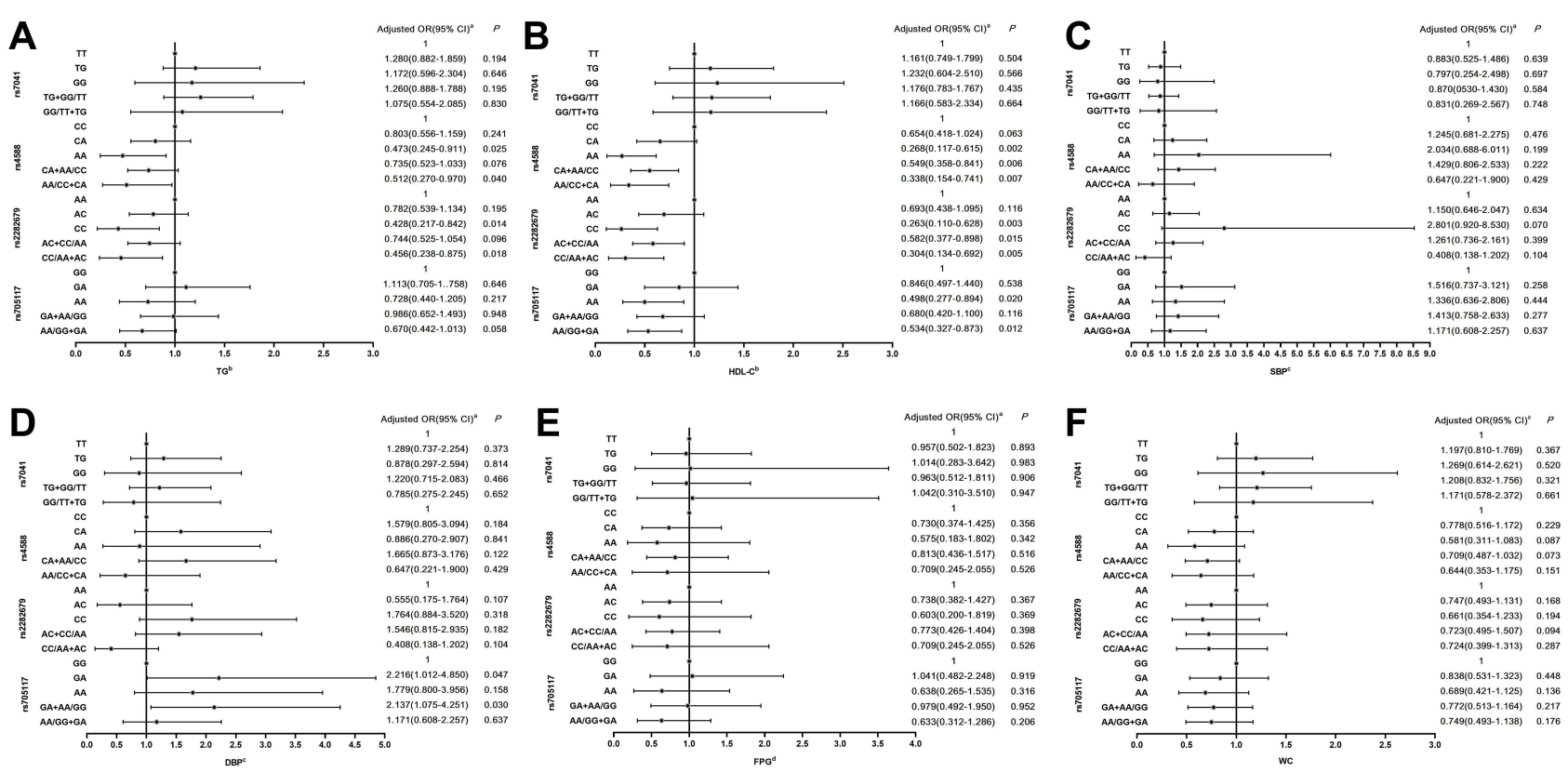

Figure 2 Association of genotypes of SNPs (rs704I, rs4588, rs2282679, and rs705I I7) of GC gene and risk of metabolic syndrome components in the rural population in Henan, China. (A) The associations between GC variants and high level of TG (triglycerides); (B) The associations between GC variants and low level of HDL-C (high-density lipoprotein-cholesterol); (C) The associations between GC variants and high level of SBP (systolic blood pressure); (D) The associations between GC variants and high level of DBP (diastolic blood pressure); (E) The associations between GC variants and high level of FPG (fasting plasma glucose); (F) The associations between GC variants and high level of WC (waist circumference).

Notes: aAdjusted for smoking status, drinking status, family history of diabetes, family history of hypertension, family history of hyperlipidemia, and family history of obesity. ${ }^{b}$ Study subjects who took lipid-lowering medicine two weeks before the survey were excluded from the statistical analysis process. 'Study subjects who took hypotensive drugs two weeks before the survey were excluded from the statistical analysis process. ${ }^{d}$ Study subjects who took anti-diabetic agents two weeks before the survey were excluded from the statistical analysis process.

In order to clarify the effect of VDBP on the interaction between $G C$ gene polymorphisms and MetS risk, we investigated the associations between $G C$ variants and VDBP

Table 4 Associations Between GC Gene and Level of VDBP

\begin{tabular}{|l|c|c|}
\hline SNP & Level of VDBP & P \\
\hline rs704I & & 0.133 \\
TT & $\mid 40.37(101.33-345.43)$ & \\
TG & $\mid 34.28(100.29-268.26)$ & \\
GG & $\mid 158.10(109.42-378.46)$ & \\
rs4588 & & 0.052 \\
CC & $\mid 49.75(103.40-361.81)$ & \\
CA & $129.86(100.05-276.37)$ & \\
AA & $153.52(106.18-336.58)$ & \\
rs2282679 & & 0.042 \\
AA & $150.91(103.92-397.11)$ & \\
AC & $129.47(100.18-276.73)$ & \\
CC & $137.08(101.33-282.53)$ & \\
rs705II7 & & 0.145 \\
GG & $151.50(103.94-423.85)$ & \\
GA & $\mid 32.14(98.81-283.09)$ & \\
AA & $\mid 43.09(105.56-282.53)$ & \\
\hline
\end{tabular}

Note: ${ }^{2}$ The level of VDBP was expressed as medians with a corresponding interquartile range. levels, as well as VDBP contents and MetS susceptibility, respectively. Based on the results, rs2282679 variants of $G C$ gene were significantly associated with changes in VDBP levels, while rs4588 variants and VDBP contents were marginally correlated. A similar result was observed in a Mendelian randomization study by Zhang et al. ${ }^{36}$ Data from a prospective cohort study in Mexico also suggested that variations in the $G C$ gene could indeed affect the expression of VDBP. ${ }^{37}$ In a cohort of Finnish men, variations in genes that are involved in the translation and posttranslational modification of $G C$ (VDBP) were shown to affect the circulating levels of VDBP. ${ }^{38}$ However, only few studies have explored the link between VDBP expression and MetS risk. Interestingly, we did not observe a clear association between VDBP and MetS risk in the study, which may be due to the relatively small sample size. Consistent with our findings, no difference was observed in the levels of VBDP between controls and patients with MetS in a caucasian population. ${ }^{39}$ A growing number of studies have demonstrated that MetS and its components are associated with the abnormal expression of vitamin $\mathrm{D},{ }^{40-42}$ and $G C$ variants was shown to influence the expression of TG, HDL-C and VDBP in this paper, so that the 
Table 5 Association and Risk Between the Level of VDBP and MetS

\begin{tabular}{|l|c|c|c|c|c|c|}
\hline Level of VDBP & Control & MetS & $\chi^{2}$ & $\boldsymbol{P}$ & OR (95\% Cl) & $\boldsymbol{P}$ \\
\hline VDBP-L & $325(73.03 \%)$ & $338(75.96 \%)$ & 2.078 & 0.354 & 1 & \\
VDBP-M & $61(13.71 \%)$ & $47(10.56 \%)$ & & & $0.735(0.505-1.122)$ & 0.163 \\
VDBP-H & $59(13.26 \%)$ & $60(13.48 \%)$ & & & $0.983(0.661-1.464)$ & 0.934 \\
\hline
\end{tabular}

Note: ${ }^{a}$ Range for levels of VDBP were defined as follows: VDBP-L $(<300 \mu \mathrm{g} / \mathrm{mL})$; VDBP-M $(300-600 \mu \mathrm{g} / \mathrm{mL}) ; \mathrm{VDBP}-\mathrm{H}(>600 \mu \mathrm{g} / \mathrm{mL})$.

dysfunctions of vitamin D-VDBP complex may contribute to the development of metabolic disorders. VDBP is an essential protein that plays a pivotal role in the transport and function of vitamin D in body, and its defects can influence the levels of circulating vitamin $\mathrm{D}$ while decreasing the production of active $1,25(\mathrm{OH})_{2} \mathrm{D}$ in targeted tissues. ${ }^{43}$ However, we did not evaluate the association among vitamin $\mathrm{D}$ and $G C$ gene and MetS risk in this paper, so further studies are required to confirm such associations.

One of the strengths of this study is that the current case-control design is done with comprehensive assessments of known and potential confounding factors. Our study is the first to systematically evaluate the relationship among $G C$ variants, VDBP expressions and MetS risks. In addition, we excluded national minority except for Han Chinese, which eliminated the complexity of genetic background. Meanwhile, some limitations of the present study should be noted. Firstly, the study was a cross-sectional study, which did not allow for the determination of causal relationships between $G C$ gene polymorphisms and MetS. Therefore, these findings should be validated using prospective studies. Secondly, SNPs located in the promoter, intron, and exon regions of the $G C$ gene were not fully included in this study, so in order to represent all variants of the GC gene, whole-gene sequencing should be required in future studies. Thirdly, we did not detect the levels of plasma vitamin D has yet been tested in our study, which may, at least partially explain the associations between $G C$ variants and MetS risk. Additionally, dietary patterns and physical activities are both closely related to the risk of MetS, ${ }^{44}$ especially the interaction for the combination of high-fat diet and low-level physical activity. Hence, further studies are needed to confirm the interactive effect of diet pattern and physical activity level on the relationships between $G C$ gene variants and MetS risk.

\section{Conclusion}

In conclusion, it was shown by our study that $G C$ polymorphism (CA+AA genotypes of rs4588 and AC genotype of rs2282679) was associated with lower metabolic syndrome susceptibility assumedly by affecting blood lipid levels in the Chinese rural population. Specific gene variants of $G C$ appear to be significant candidates predisposing to MetS in the Chinese rural population. Nevertheless, these results need to be confirmed whether these associations are ethnic specificity.

\section{Abbreviations}

IDF, International Diabetes Federation; VDBP, vitamin D binding protein; GC, group-specific component globulin; MetS, metabolic syndrome; OR, odds ratio; SNP, single nucleotide polymorphism; HWE, Hardy-Weinberg equilibrium; T2DM, type 2 diabetes mellitus; HDL-C, highdensity lipoprotein-cholesterol; LDL-C, low-density lipoprotein cholesterol; TG, triglycerides; BP, blood pressure; SBP, systolic blood pressure; DBP, diastolic blood pressure; FPG, fasting plasma glucose; HC, high cholesterol, MAF, minor allele frequency; 25(OH)D, 25-hydroxyvitamin D; WC, waist circumference; BMI, body mass index.

\section{Data Sharing Statement}

All data generated or analyzed during this study are included in this article. Further enquiries can be directed to the corresponding author.

\section{Ethics Approval and Informed Consent}

Written informed consent was obtained from each participant. The study was conducted with the approval from the Ethics Committee of Zhengzhou University (Code: [2015] MEC (S128)), and adhere to the tenets of the Declaration of Helsinki.

\section{Consent for Publication}

All the authors agreed to the publication statements.

\section{Acknowledgments}

We would like to acknowledge all subjects included in this study for their participation. 


\section{Funding}

This work was supported by the National Natural Science Foundation of China (grant numbers 81872626 and 82003454), Chinese N utrition Society-Bright Moon Seaweed Group Nutrition and Health Research Fund (grant number CNS-BMSG2020A63), Chinese Nutrition Society-Zhendong National Physical Fitness and Health Research Fund (grant number CNS-ZD2019066), Key R\&D and promotion projects in Henan Province (grant number 212102310219). All the funders had no role in the design, analysis, or writing of this article.

\section{Disclosure}

The authors declare no conflicts of interest.

\section{References}

1. Alberti KG, Zimmet P, Shaw J. The metabolic syndrome-a new worldwide definition. Lancet. 2005;366(9491):1059-1062. doi:10. 1016/s0140-6736(05)67402-8

2. Alberti G, Zimmet P. The IDF consensus worldwide definition of the metabolic syndrome. 2006.

3. Stern MP, Williams K, González-Villalpando C, Hunt KJ, Haffner SM. Does the metabolic syndrome improve identification of individuals at risk of type 2 diabetes and/or cardiovascular disease? Diabetes Care. 2004;27(11):2676-2681. doi:10.2337/ diacare.27.11.2676

4. Group DIS. Plasma insulin and cardiovascular mortality in non-diabetic European men and women: a meta-analysis of data from eleven prospective studies. Diabetologia. 2004;47(7): 1245-1256. doi:10.1007/s00125-004-1433-4

5. Carr DB, Utzschneider KM, Hull RL, et al. Intra-abdominal fat is a major determinant of the National Cholesterol Education Program Adult Treatment Panel III criteria for the metabolic syndrome. Diabetes. 2004;53(8):2087-2094. doi:10.2337/diabetes.53.8.2087

6. Prasad G, Bandesh K, Giri AK, et al. Genome-wide association study of metabolic syndrome reveals primary genetic variants at CETP locus in Indians. Biomolecules. 2019;9(8):321. doi:10.3390/biom9080321

7. Melguizo-Rodriguez L, Costela-Ruiz VJ, Garcia-Recio E, De Lunabertos E, Ruiz C, Illescas-Montes R. Role of vitamin D in the metabolic syndrome. Nutrients. 2021;13(3):830. doi:10.3390/nu 13030830

8. Schmitt EB, Nahas-Neto J, Bueloni-Dias F, Poloni PF, Orsatti CL, Petri Nahas EA. Vitamin D deficiency is associated with metabolic syndrome in postmenopausal women. Maturitas. 2018;107:97-102. doi:10.1016/j.maturitas.2017.10.011

9. Xiao J, Lv J, Wang S, et al. Association of serum 25-hydroxyvitamin D with metabolic syndrome and type 2 diabetes: a one sample Mendelian randomization study. BMC Geriatr. 2021;21(1):391. doi:10.1186/s12877-021-02307-6

10. Erdman JW, Macdonald IA, Zeisel SH. Present Knowl Nutr. John Wiley \& Sons; 2012.

11. Jiang X, O'Reilly PF, Aschard H, et al. Genome-wide association study in 79,366 European-ancestry individuals informs the genetic architecture of 25-hydroxyvitamin D levels. Nat Commun. 2018;9 (1):260. doi:10.1038/s41467-017-02662-2

12. Delanghe JR, Speeckaert R, Speeckaert MM. Behind the scenes of vitamin D binding protein: more than vitamin D binding. Best Pract Res Clin Endocrinol Metab. 2015;29(5):773-786. doi:10.1016/j. beem.2015.06.006
13. Saad MF, Lillioja S, Nyomba BL, et al. Racial differences in the relation between blood pressure and insulin resistance. $N$ Engl J Med. 1991;324(11):733-739. doi:10.1056/NEJM199103143241105

14. Choi J, Choi JY, Lee SA, et al. Association between family history of diabetes and clusters of adherence to healthy behaviors: cross-sectional results from the Health Examinees-Gem (HEXA-G) study. BMJ Open. 2019;9(6):e025477. doi:10.1136/bmjopen-2018025477

15. Wang C, Li L, Wang L, et al. Evaluating the risk of type 2 diabetes mellitus using artificial neural network: an effective classification approach. Diabetes Res Clin Pract. 2013;100(1):111-118. doi:10.1016/j.diabres.2013.01.023

16. Weng J, Ji L, Jia W, et al. Standards of care for type 2 diabetes in China. Diabetes Metab Res Rev. 2016;32(5):442-458. doi:10.1002/ dmrr.2827

17. Friedewald WT, Levy RI, Fredrickson DS. Estimation of the concentration of low-density lipoprotein cholesterol in plasma, without use of the preparative ultracentrifuge. Clin Chem. 1972;18(6):499-502. doi:10.1093/clinchem/18.6.499

18. Rahimi MH, Mollahosseini M, Mirzababaei A, Yekaninejad MS, Maghbooli Z, Mirzaei K. Interactions between vitamin D binding protein variants and major dietary patterns on the odds of metabolic syndrome and its components in apparently healthy adults. Diabetol Metab Syndr. 2019;11:28. doi:10.1186/s13098-019-0422-1

19. Karuwanarint P, Phonrat B, Tungtrongchitr A, et al. Vitamin D-binding protein and its polymorphisms as a predictor for metabolic syndrome. Biomark Med. 2018;12(5):465-473. doi:10.2217/bmm2018-0029

20. Kawakami M, Blum CB, Ramakrishnan R, Dell RB, Goodman DS. Turnover of the plasma binding protein for vitamin D and its metabolites in normal human subjects. J Clin Endocrinol Metab. 1981;53 (6):1110-1116. doi:10.1210/jcem-53-6-1110

21. Cornier MA, Dabelea D, Hernandez TL, et al. The metabolic syndrome. Endocr Rev. 2008;29(7):777-822. doi:10.1210/er.20080024

22. Mehramiz M, Khayyatzadeh SS, Esmaily H, et al. Associations of vitamin $\mathrm{D}$ binding protein variants with the vitamin D-induced increase in serum 25-hydroxyvitamin D. Clin Nutr ESPEN. 2019;29:59-64. doi:10.1016/j.clnesp.2018.12.005

23. Bahrami A, Sadeghnia HR, Tabatabaeizadeh SA, et al. Genetic and epigenetic factors influencing vitamin D status. J Cell Physiol. 2018;233(5):4033-4043. doi:10.1002/jcp.26216

24. Karonova T, Grineva E, Belyaeva O, et al. Relationship between vitamin D status and vitamin D receptor gene polymorphisms with markers of metabolic syndrome among adults. Front Endocrinol (Lausanne). 2018;9:448. doi:10.3389/fendo.2018.00448

25. Baralle D, Baralle M. Splicing in action: assessing disease causing sequence changes. J Med Genet. 2005;42(10):737-748. doi:10.1136/ jmg.2004.029538

26. Grave N, Tovo-Rodrigues L, da Silveira J, et al. A vitamin D pathway gene-gene interaction affects low-density lipoprotein cholesterol levels. J Nutr Biochem. 2016;38:12-17. doi:10.1016/j.jnutbio. 2016.08.002

27. Foucan L, Vélayoudom-Céphise FL, Larifla L, et al. Polymorphisms in GC and NADSYN1 genes are associated with vitamin D status and metabolic profile in non-diabetic adults. BMC Endocr Disord. 2013;13:36. doi:10.1186/1472-6823-13-36

28. Bagnasco NJT, Moreira ARS, Martins M, Farias D, Kac G. Effects of the polymorphisms rs17782313 in mc4r gene and rs2282679 in VDBP gene on the TG-HDL ratio and HOMA-IR through pregnancy. Diabetol Metab Syndr. 2015;7(S1). doi:10.1186/17585996-7-s1-a223

29. Scicali R, Giral P, D'Erasmo L, et al. High TG to HDL ratio plays a significant role on atherosclerosis extension in prediabetes and newly diagnosed type 2 diabetes subjects. Diabetes Metab Res Rev. 2021;37(2):e3367. doi:10.1002/dmrr.3367 
30. Otsuka T, Takada H, Nishiyama Y, et al. Dyslipidemia and the risk of developing hypertension in a working-age male population. $J$ Am Heart Assoc. 2016;5(3):e003053. doi:10.1161/jaha.115.00 3053

31. Vekic J, Zeljkovic A, Stefanovic A, Jelic-Ivanovic Z, SpasojevicKalimanovska V. Obesity and dyslipidemia. Metabolism. 2019;92:71-81. doi:10.1016/j.metabol.2018.11.005

32. Sesso HD, Buring JE, Chown MJ, Ridker PM, Gaziano JM. A prospective study of plasma lipid levels and hypertension in women. Arch Intern Med. 2005;165(20):2420-2427. doi:10.1001/ archinte. 165.20.2420

33. Cochran BJ, Ong KL, Manandhar B, Rye KA. High density lipoproteins and diabetes. Cells. 2021;10(4):850. doi:10.3390/cells10040850

34. Stadler JT, Marsche G. Obesity-related changes in high-density lipoprotein metabolism and function. Int J Mol Sci. 2020;21(23):8985. doi:10.3390/ijms21238985

35. Blaton V. How is the metabolic syndrome related to the dyslipidemia? Ejifcc. 2007;18(1):15-22.

36. Zhang $\mathrm{H}$, Wang $\mathrm{T}$, Han $\mathrm{Z}$, et al. Impact of vitamin $\mathrm{D}$ binding protein levels on Alzheimer's disease: a Mendelian randomization study. J Alzheimers Dis. 2020;74(3):991-998. doi:10.3233/jad-191051

37. Rivera-Paredez B, Hidalgo-Bravo A, León-Reyes G, et al. Association of GC variants with bone mineral density and serum VDBP concentrations in Mexican population. Genes. 2021;12 (8):1176. doi:10.3390/genes 12081176
38. Moy KA, Mondul AM, Zhang $\mathrm{H}$, et al. Genome-wide association study of circulating vitamin D-binding protein. Am J Clin Nutr. 2014;99(6):1424-1431. doi:10.3945/ajcn.113.080309

39. Pelczynska M, Grzelak T, Sperling M, Bogdanski P, Pupek-Musialik D, Czyzewska K. Impact of 25-hydroxyvitamin D, free and bioavailable fractions of vitamin $\mathrm{D}$, and vitamin $\mathrm{D}$ binding protein levels on metabolic syndrome components. Arch Med Sci. 2017;13 (4):745-752. doi:10.5114/aoms.2016.58594

40. Vaidya A, Forman JP. Vitamin D and hypertension: current evidence and future directions. Hypertension. 2010;56(5):774-779. doi:10.1161/HYPERTENSIONAHA.109.140160

41. Takiishi T, Gysemans C, Bouillon R, Mathieu C. Vitamin D and diabetes. Endocrinol Metab Clin North Am. 2010;39(2):419-46, table of contents. doi:10.1016/j.ecl.2010.02.013

42. Pourshahidi LK. Vitamin D and obesity: current perspectives and future directions. Proc Nutr Soc. 2015;74(2):115-124. doi:10.1017/ s0029665114001578

43. Almesri N, Das NS, Ali ME, Gumaa K, Giha HA. Independent associations of polymorphisms in vitamin D binding protein (GC) and vitamin D receptor (VDR) genes with obesity and plasma 25OHD3 levels demonstrate sex dimorphism. Appl Physiol Nutr Metabol. 2016;41(4):345-353. doi:10.1139/apnm-2015-0284

44. De Sousa SMD, Norman RJP. Metabolic syndrome, diet and exercise. Best Pract Res Clin Obstet Gynaecol. 2016;37:140-151. doi:10.1016/ j.bpobgyn.2016.01.006

\section{Publish your work in this journal}

Diabetes, Metabolic Syndrome and Obesity: Targets and Therapy is an international, peer-reviewed open-access journal committed to the rapid publication of the latest laboratory and clinical findings in the fields of diabetes, metabolic syndrome and obesity research. Original research, review, case reports, hypothesis formation, expert opinion and commentaries are all considered for publication. The manuscript management system is completely online and includes a very quick and fair peer-review system, which is all easy to use. Visit http://www.dovepress.com/testimonials.php to read real quotes from published authors.

Submit your manuscript here: https://www.dovepress.com/diabetes-metabolic-syndrome-and-obesity-targets-and-therapy-journal 